\title{
THE WEAK BEHAVIOR OF SPHERICAL MEANS
}

\author{
CARLOS E. KENIG AND PETER A. TOMAS ${ }^{1}$
}

\begin{abstract}
C. Fefferman has shown that the disc multiplier is not bounded on $L^{p}\left(\mathbf{R}^{n}\right), n>1, p \neq 2$. In contrast, $\mathbf{C}$. Herz showed that, when restricted to $L^{p}$ radial functions, it is bounded on $L^{p}\left(\mathbf{R}^{n}\right)$ if and only if $2 n /(n+1)<$ $p<2 n /(n-1)$. We show that it is not weakly bounded for $p=2 n /(n+1)$ or $p=2 n /(n-1)$.
\end{abstract}

Define the linear operator $T$ on $L^{2}\left(\mathbf{R}^{n}\right)$ by

$$
\widehat{T f}(y)= \begin{cases}\hat{f}(y) & \text { if }|y|<1 \\ 0 & \text { if }|y|>1\end{cases}
$$

We shall assume throughout the remainder of this paper that $n>1$. Charles Fefferman [1] has shown that $T$ is not bounded on $L^{p}\left(\mathbf{R}^{n}\right)$ for any $p \neq 2$. In contrast, Herz [2] showed that if one is concerned only with $L^{p}$ radial functions, $T$ is bounded on $L^{p}\left(\mathbf{R}^{n}\right)$ if and only if $2 n /(n+1)<p<2 n /(n-$ 1). We shall establish the following result.

THEOREM. $T$ is not weakly bounded on $L^{p}\left(\mathbf{R}^{n}\right)$ radial functions for $p=2 n /(n$ $+1)$.

The proof is quite simple. Following Herz, note first that if $f$ is a radial function on $\mathbf{R}^{n}, \hat{f}$ is also radial, and

$$
\hat{f}(R)=2 \pi \int_{0}^{\infty} \frac{J_{(n-2) / 2}(2 \pi r R)}{|r R|^{(n-2) / 2}} f(r) r^{n-1} d r .
$$

Therefore

$$
T f(s)=\frac{4 \pi^{2}}{s^{(n-2) / 2}} \int_{0}^{\infty} r^{n / 2} f(r) \int_{0}^{1} J_{(n-2) / 2}(2 \pi s R) J_{(n-2) / 2}(2 \pi r R) R d R d r .
$$

Denote the inner integral by $K(s, r)$. From Watson [3, p. 46], we see

$$
\left(x^{k} J_{k}(x)\right)^{\prime}=x^{k} J_{k-1}(x), \quad\left(x^{-k} J_{k}(x)\right)^{\prime}=-x^{-k} J_{k+1}(x) .
$$

Employing these in an integration by parts in the expression for $K$, we see $2 \pi K(s, r)=\frac{1}{r} J_{(n-2) / 2}(2 \pi s) J_{n / 2}(2 \pi r)+2 \pi \frac{s}{r} \int_{0}^{1} J_{n / 2}(2 \pi s R) J_{n / 2}(2 \pi r R) R d R$.

Noting the symmetry of $K$ in $r$ and $s$, we compute $2 \pi K(s, r)(r / s-s / r)$, and

Received by the editors October 5, 1977.

AMS (MOS) subject classifications (1970). Primary 42A18.

Key words and phrases. Disc multiplier on radial functions, failure of weak type estimates.

${ }^{1}$ The first named author is a Victor J. Andrew Fellow at the University of Chicago; the second named author was supported in part by the National Science Foundation. 
obtain from this the result

$$
\begin{aligned}
T f(s)= & \frac{2 \pi}{s^{(n-2) / 2}} \int_{0}^{\infty} s \frac{J_{(n-2) / 2}(2 \pi r) J_{n / 2}(2 \pi s)}{s^{2}-r^{2}} f(r) r^{n / 2} d r \\
& -\frac{2 \pi}{s^{(n-2) / 2}} \int_{0}^{\infty} r \frac{J_{(n-2) / 2}(2 \pi s) J_{n / 2}(2 \pi r)}{s^{2}-r^{2}} f(r) r^{n / 2} d r \\
= & T_{0} f(s)+T_{1} f(s) .
\end{aligned}
$$

We shall let

$$
f(r)=\frac{J_{(n-2) / 2}(2 \pi r)}{r^{n / 2}} \chi_{(1, \beta)}(r)
$$

for large $\beta$, and shall evaluate $T f(s)$ for $s>2 \beta$. On such functions, $T_{1}$ is an operator which is weakly bounded:

$$
\left|T_{1} f(s)\right| \leqslant c \frac{\left|J_{(n-2) / 2}(2 \pi s)\right|}{s^{(n-2) / 2}} \int_{0}^{s / 2} \frac{\left|J_{n / 2}(2 \pi r)\right|}{s^{2}-r^{2}} r f(r) r^{n / 2} d r .
$$

Using the asymptotic relationships

$$
\begin{aligned}
J_{k}(r) & =\left(\frac{2}{\pi r}\right)^{1 / 2} \cos \left(r-\frac{\pi k}{2}-\frac{\pi}{4}\right)+o\left(\frac{1}{r^{3 / 2}}\right), \quad r \rightarrow \infty, \\
& \sim c r^{k}, \quad r \rightarrow 0,
\end{aligned}
$$

we see that

$$
\begin{aligned}
\left|T_{1} f(s)\right| & <\frac{c}{s^{(n+1) / 2} s}\left(\int_{0}^{s / 2}\left|\frac{J_{n / 2}(2 \pi r)}{r^{n / 2}} r^{2}\right|^{2 n /(n-1)} r^{n-1} d r\right)^{(n-1) / 2 n}\|f\|_{2 n /(n+1)} \\
& <\frac{c\|f\|_{2 n /(n+1)}}{s^{(n+1) / 2}}
\end{aligned}
$$

Thus $T_{1}$ is weakly bounded at $2 n /(n+1)$, with respect to the measure $d \mu=r^{n-1} d r$, and to prove the result it suffices to show $T_{0}$ is not. But for the given $f$, the asymptotics for the Bessel functions show that for $s>2 \beta$,

$$
\begin{aligned}
\left|T_{0} f(s)\right| & =c_{0} \frac{\left|J_{n / 2}(2 \pi s)\right|}{s^{(n-2) / 2}} \int_{1}^{\beta}\left|J_{(n-2) / 2}(2 \pi r)\right|^{2} \frac{s}{s^{2}-r^{2}} d r \\
& \geqslant c_{1} \frac{\left|J_{n / 2}(2 \pi s)\right|}{s^{n / 2}} \int_{1}^{\beta} \frac{|\cos (2 \pi r-\pi(n-3) / 4)|^{2}}{r} d r \\
& \geqslant c_{2} \frac{\left|J_{n / 2}(2 \pi s)\right|}{s^{n / 2}} \log \beta .
\end{aligned}
$$

Therefore for $\beta$ large,

$$
\mu\left\{s \geqslant 2 \beta|| T_{0} f(s) \mid>\alpha\right\} \geqslant c_{3} \mu\left\{s>2 \beta \mid c_{2} \frac{\log \beta}{\alpha}>s^{(n+1) / 2}\right\} .
$$

Choose $\alpha=(\log \beta) / c_{4} \beta^{(n+1) / 2}$; then

$$
\mu\left\{s|| T_{0} f(s) \mid>\alpha\right\} \geqslant c_{3} \int_{2 \beta}^{c_{5} \beta} s^{n-1} d s,
$$


where $c_{5}=\left(c_{2} c_{4}\right)^{2 /(n+1)}$ is chosen so that $\left(c_{5}^{n}-2^{n}\right) c_{3} / n=1$. Thus, all in all,

$$
\mu\left\{s|| T_{0} f(s) \mid>\alpha\right\} \geqslant \beta^{n} \text {. }
$$

But if $T_{0}$ is weakly bounded,

$$
\begin{aligned}
\beta^{n} & <\mu\left\{s|| T_{0} f(s) \mid>\alpha\right\} \leqslant c_{6}\left(\frac{\|f\|_{2 n /(n+1)}}{\alpha}\right)^{2 n /(n+1)} \\
& <c_{7} \log \beta\left(\frac{\log \beta}{c_{4} \beta^{(n+1) / 2}}\right)^{-2 n /(n+1)}=c_{8} \beta^{n}(\log \beta)^{-(n-1) /(n+1)} .
\end{aligned}
$$

This is a contradiction.

The same counterexample can be used to show that $T$ is not weakly bounded on $L^{p}\left(\mathbf{R}^{n}\right)$ if $p=2 n /(n-1)$, by showing the adjoint of $T$ is not bounded from $L^{p^{\prime}, 1}$ to $L^{p^{\prime}}$.

\section{REFERENCES}

1. C. Fefferman, The multiplier problem for the ball, Ann. of Math. 94 (1971), 330-336.

2. C. Herz, On the mean inversion of Fourier and Hankel transforms, Proc. Nat. Acad. Sci. U.S.A. 40 (1954), 996-999.

3. G. N. Watson, Theory of Bessel functions, Cambridge Univ. Press, London, 1966.

Department of Mathematics, Princeton University, Princeton, New Jersey 08540

Department of Mathematics, University of TeXas, Austin, TeXas 78712 